\title{
An appliance using short skeletal anchorage screws to support the movement of impacted cuspids into the arch
}

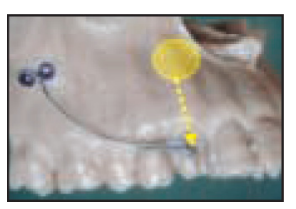

\section{Daniel CHILLÈS, Jean-Gabriel CHILLÈS}

\begin{abstract}
This article shows how to apply orthodontie force to impacted cuspids and bring them into proper arch position using a unique innovative anchorage system consisting of two short mini-screws placed buccally in cortical bone. Attached to this fixed anchorage is an .018 TMA round wire that emerges from the gingiva to exert direct $3 D$ control on the canine without any additional dental support. The general procedure for moving impacted teeth into position and the mechanics utilized are described in detail with illustrations from clinical cases we have treated.
\end{abstract}

\section{KEYWORDS}

Orthodontics

Impacted canine

Bone screws

Mini-screws

Short cortical screw. 


\section{1 - INTRODUCTION}

When traditional anchorage based on dental units is insufficient for correction of a deformity, when the practitioner wants to simplify orthodontic mechanics or diminish, even eliminate, anchorage based on teeth, it is possible today to rely on anchorage set in bone $1,3,4,7,9,10,11$.

The principal defect of long transmucosal bone screws is that they need to be supported by sufficient bone beneath attached gingiva9,11 and if they pass through spongy bone it is essential that they do not impact upon any adjacent underlying anatomic structures ${ }^{2}$. What is perhaps worse, they are relatively unreliable $e^{8,12}$, a deficiency which, in most cases, limits them to subordinate roles.

And osseous anchorage plates require surgical procedures that only trained oral surgeons can perform for their installation. This added complication, the not infrequent postoperative problems that affect them, and their relatively high cost strongly curtail their indications $s^{1,7,13}$.

After considerable experience with trans-mucosal screws ${ }^{3,4}$ we experimented with and developed the technique of using microscrews to pin arch wires directly against cortical

\section{2 - CHARACTERISTICS OF THE MATERIALS}

\section{2 - 1 - The screws}

We use $5 \mathrm{~mm}$ long self-tapping bone screws that are $2 \mathrm{~mm}$ in diameter and designed to be embedded in bone. Their relatively diminutive size makes them far less obtrusive beneath the mucosa than other devices (fig. 1 b). bone $e^{3,4}$. We place them under the mucosa at the depth of the vestibule. The surgical procedure is simple, requiring neither flap nor sutures, with very few post-operative complications. Orthodontists can readily screw them in place themselves ${ }^{2}$.

If we insert two screws side by side and use an arch wire that is both extremely flexible and adjustable like steel or TMA 6 (fig. 1a), we can, from a distance, exert forces in all three dimensions of space. The system becomes totally autonomous and polyvalent.

We shall show that this technique is perfectly adapted to moving impacted cuspids into place in a greatly simplified procedure that makes incorporating other teeth into the anchorage system unnecessary and thus avoids any unwelcome parasitic dental movements.

After having explained the specific implementation of this system as well as its advantages compared to other bone anchorage techniques, we shall review the operative procedure and the mechanics we use in detail and illustrate them fully with representative clinical cases treated in our office.

In contrast to the trans-mucosal screws, this short variety goes no further than underlying cortical bone and does not penetrate into spongy bone regions. So, because the arch wire, being braced against the bone itself, does not act like a lever arm that might dislodge the screws that bind it, their primary stability is excellent (fig. 1 c). 
In spite of their small size, the screws' $2 \mathrm{~mm}$ diameter offers sufficient contact surface area for correct retention and mechanics.

They are made of a titanium alloy $\mathrm{TiAl}_{6}-\mathrm{V}_{4}$ (norm ISO 5832-3 and ASTM F136), which renders them bio-compatible without encouraging osteointegration which would make their removal after the completion of treatment difficult ${ }^{4}$.

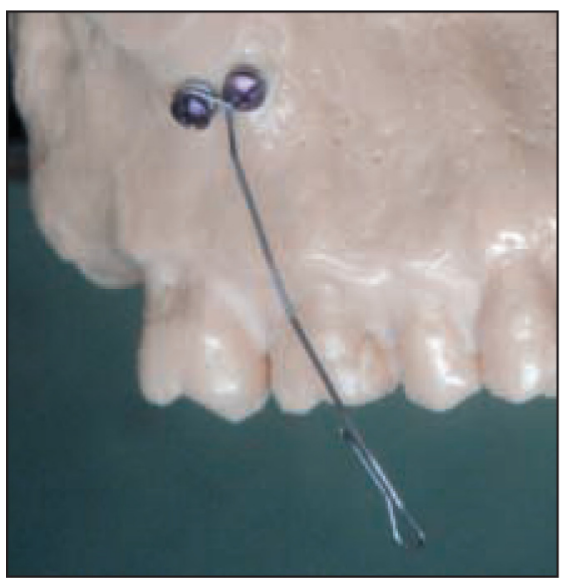

Figure 1 a

Two screws are placed side by side with a wire that is both malleable and flexible.
Because of their shortness, they pose a reduced risk to nearby anatomical structures and can be placed with little fear of their being obtrusive almost anywhere beneath the mucosa (fig. 1 d), except, of course near the mental foramen in cases of impacted lower canines, an advantage that is not share by long trans-mucosal screws. We have found that the most favorable sites for providing anchorage for

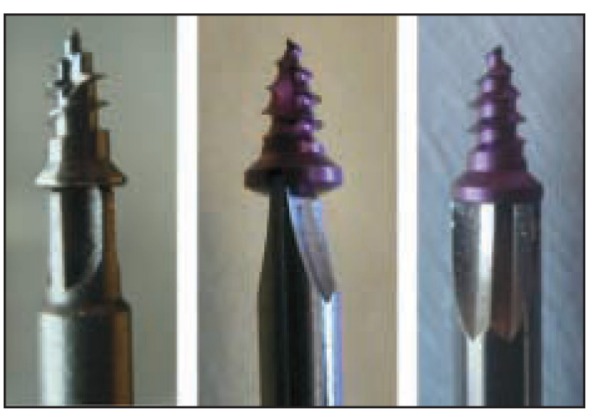

Figure $1 \mathrm{~b}$

Five $\mathrm{mm}$ long, two $\mathrm{mm}$ wide self tapping bone screws destined to be embedded. The thinness of their heads prevents them from being a bulky sub-mucosal intrusion. They can reliably be carried to their destinations at the end of the screwdriver.

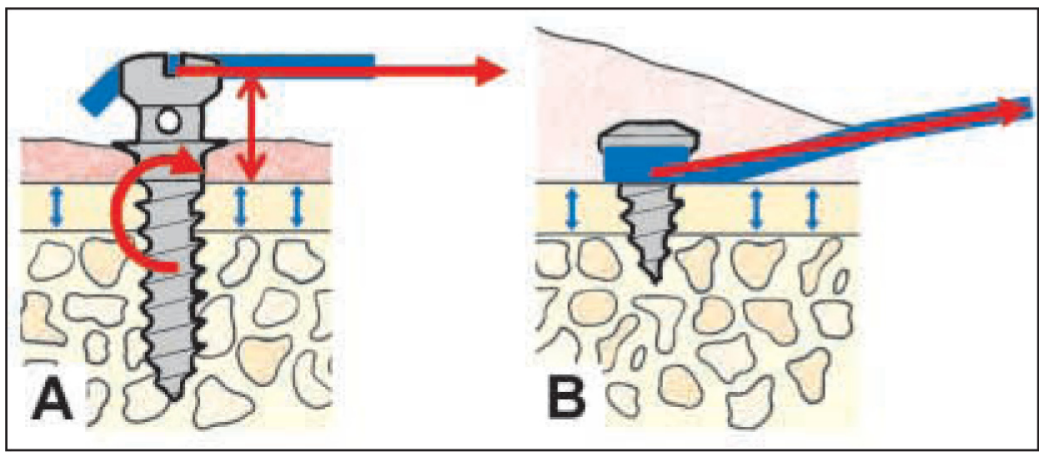

Figure $1 \mathrm{C}$

In contrast to trans-mucosal screws $(A)$, these short screws are subject to practically no lever arm force and so resist constraints tending to remove them $(B)$. 


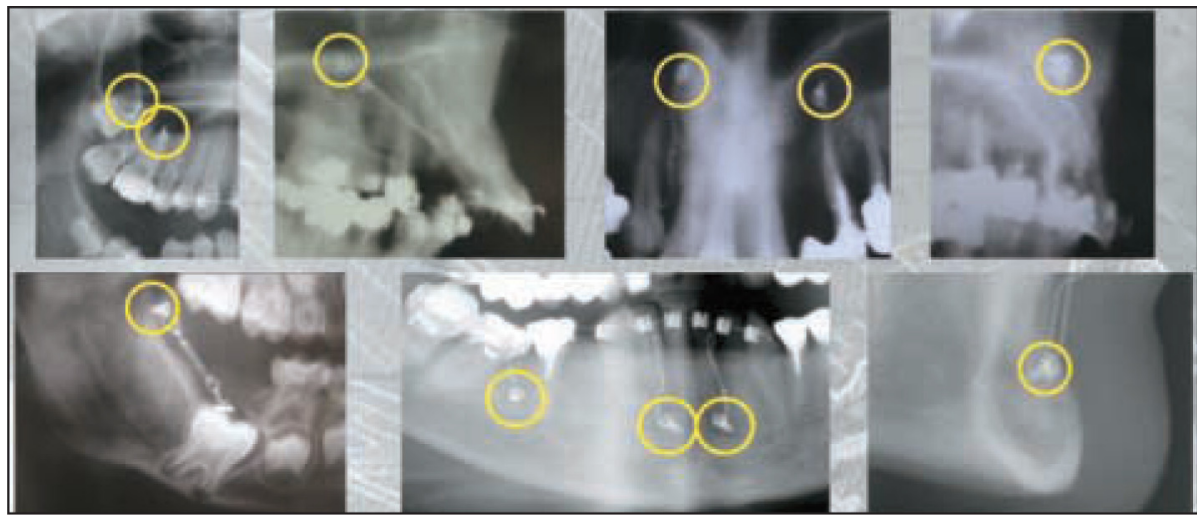

Figure $1 d$

The short cortical screws can be placed almost anywhere beneath the mucosa.

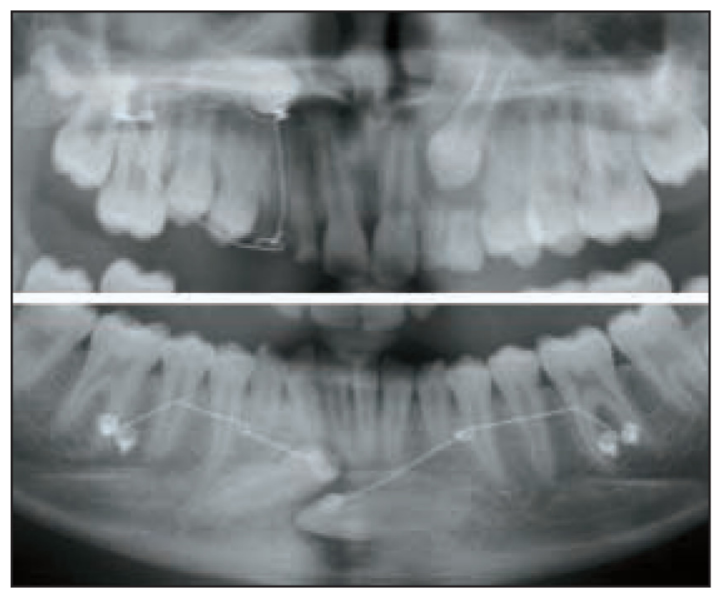

Figure 1 e

To support the force applied to impacted canines, we position the bone screws at the level of the first molars.

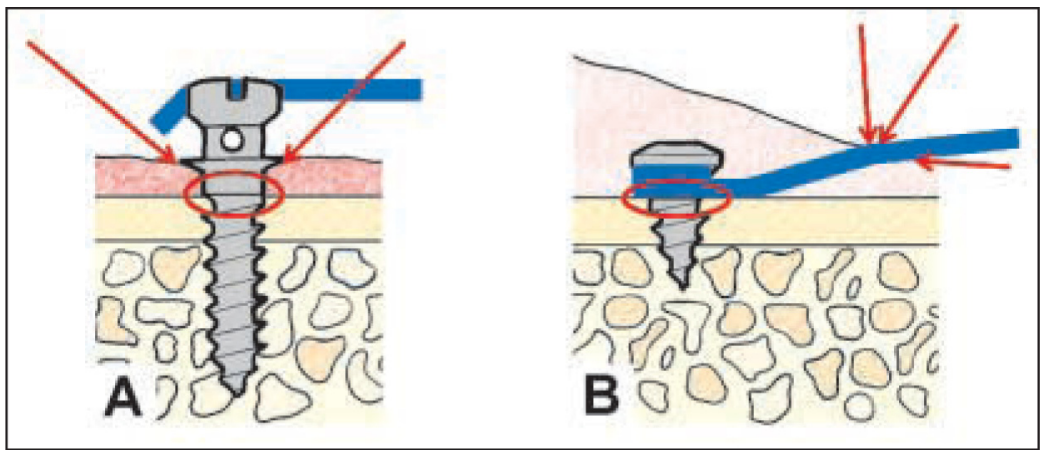

Figure $1 f$

In contrast to trans-mucosal screws (A), the short distance from the sub-mucosal position of the short screw heads to the emergence of the wire eliminates the risk of bacterial infiltration into the bone (B). 
movement of impacted canines are zones situated beneath the apices of the first molars (fig. 1e). In the maxilla this puts them in the zygomatic process that can readily be located by palpation and which, according to all authorities, assures the presence of good quality bone $1,3,79,11$.

Their self-tapping character and the absence of any anatomic risk makes it possible for us to put the short screws in place with very little displacement of mucosa, without drilling a preparatory opening, even when we work without good visual access, and without, almost ever, incurring any post-operative sequelae, which is certainly not the case with embedded osseous plates. They do share the plates' quality of not limiting the amplitude of possible movement of teeth.

In contrast with trans-mucosal screws, the possibility of loosening of the short screws is negligible because the distance between their heads, under the mucosa, and the emergence of the wire is great enough to virtually eliminate the risk of bacterial infiltration into bone (fig. $1 \mathrm{f}$ ).

We can, accordingly, place these screws in the depth of the buccal cavity because they do not have to be in a region of attached gingiva, a placement traditionally considered crucial for reducing the risk of an infection that would cause loss of the screws $s^{1,3,8,12}$.

Because of this, our system is extremely reliable and to this date not one of the more than 150 short screws we have placed in our office has been lost under normal conditions.

They are self prehensile, which means that we can seize them with the screwdriver and they remain there, securely in place at the end of this tool (fig. 1 b).

And when it becomes time for removal, we can find the heads of the screws easily, even without visual access. Usually they stick to the screwdriver after being unscrewed.

\section{2 - 2 - The wires}

We have tested steel wires successfully but today prefer to use $0.45 \mathrm{~mm}$ (.018) TMA wires, which seem to us to be more reliable.

We call it CT8-1 (Cortical TMA, ending in a figure "8" form, one arm) in our classification ${ }^{5}$ that includes four types differentiated by the number of directional controls they afford. Here it is "3D".

The terminal double figure "8" shape allows us to affix the wire with two screws placed side by side, leaving its activated long free arm ready to deliver spring force action (fig. 2 a).

Because of its extreme flexibility, the wire does not transmit, during treatment, excessive muscular forces directly to the anchorage system as happens with embedded plates, where breakage ${ }^{14}$ or loosened screws ${ }^{1}$ can cause loss of the entire assemblage.

We begin at the end of an approximately $11 \mathrm{~cm}$ wire by shaping two figure "8" loops (fig. 5 a) that will receive the bone screws (fig. 2 b).

The residual length of the wire of about $9 \mathrm{~cm}$ will be great enough so that the handle of the screwdriver can direct it as it is being put in place (fig. 2 c).

The double figure "8" loops make it possible for us to position the wire firmly against the bone and have 


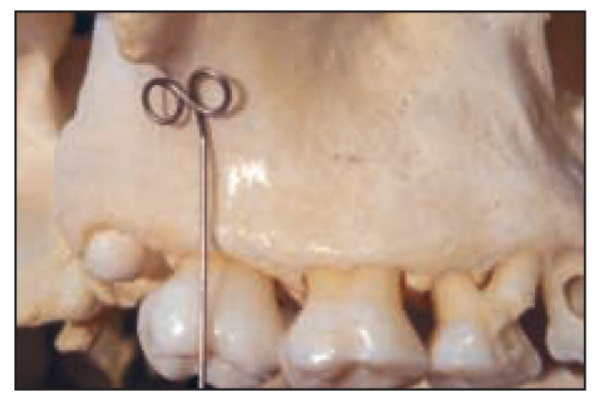

Figure 2 a

CT8-1 (Cortical TMA, terminating in double figure "8" shape, one arm). This double figure " 8 " permits fixation of the long activated arm to the bone with two screws set side by side.

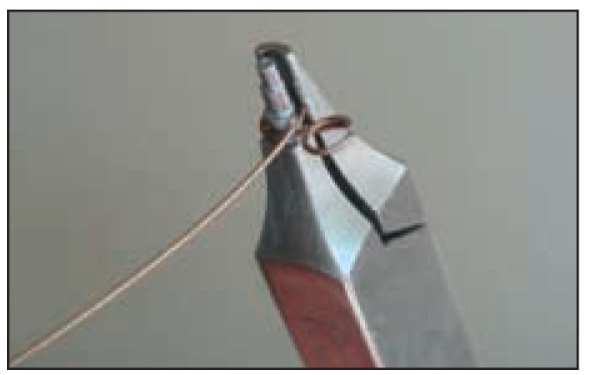

Figure $2 b$

The two figure "8" loops are bent with pliers at the end of a straight wire about $11 \mathrm{~cm}$ long.

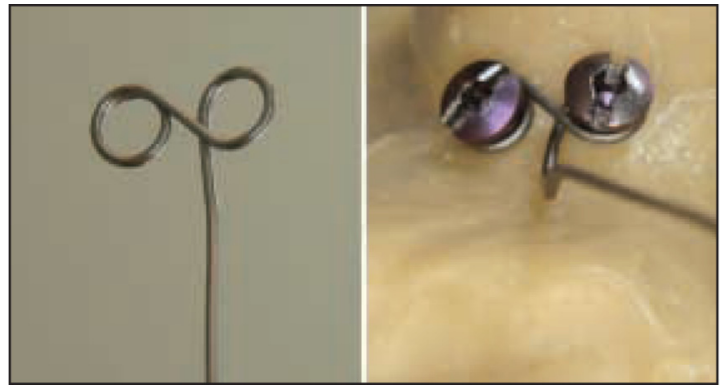

Figure $2 d$

Design for the loops on the right side (for the maxilla). The active arm of is pressed against bone by the cross sector of the wire between the two screws. As the free arm is activated the pressure around the mesial screw will be reinforced.

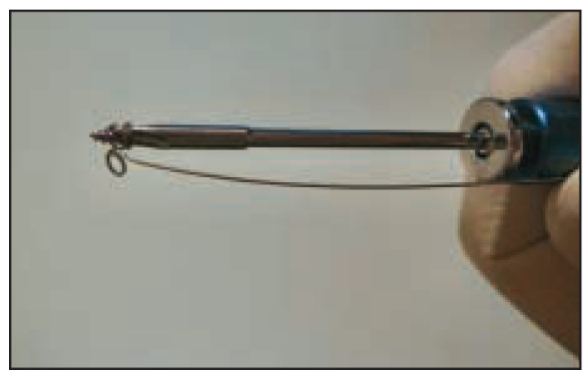

Figure $2 \mathrm{c}$

Using finger pressure the orthodontist holds the end of the wire against the handle of the screwdriver, which must be long enough to prevent the patient's cheeks from interfering with its manipulation.

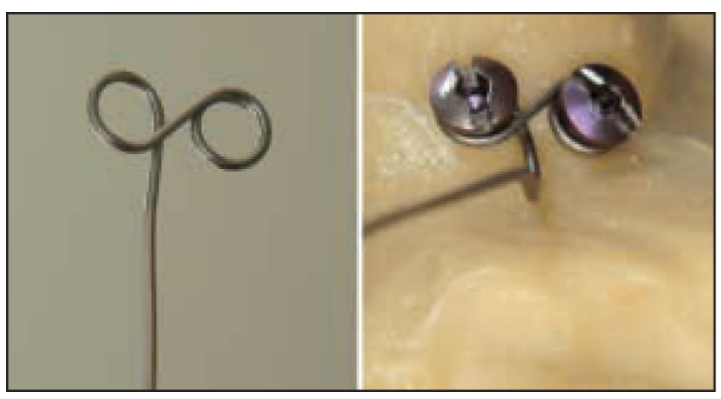

Figure $2 \mathrm{e}$

Design for the loops on the left (for the maxilla). The active arm is pressed against the bone as in the previous figure. 


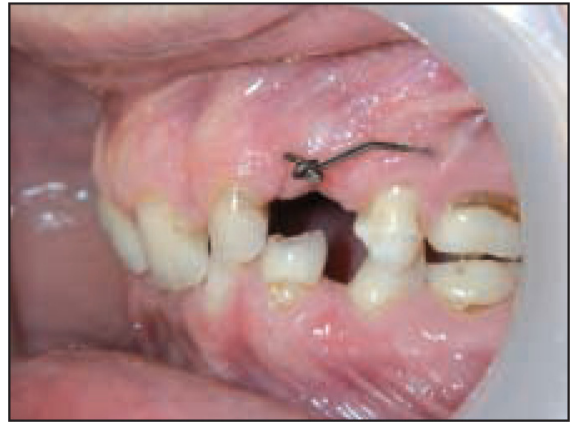

Figure $2 f$

Palatally impacted canine of a patient many of whose teeth were congenitally absent. The wire, which emerges at the muco-gingival line, is applying buccal force to the tooth.

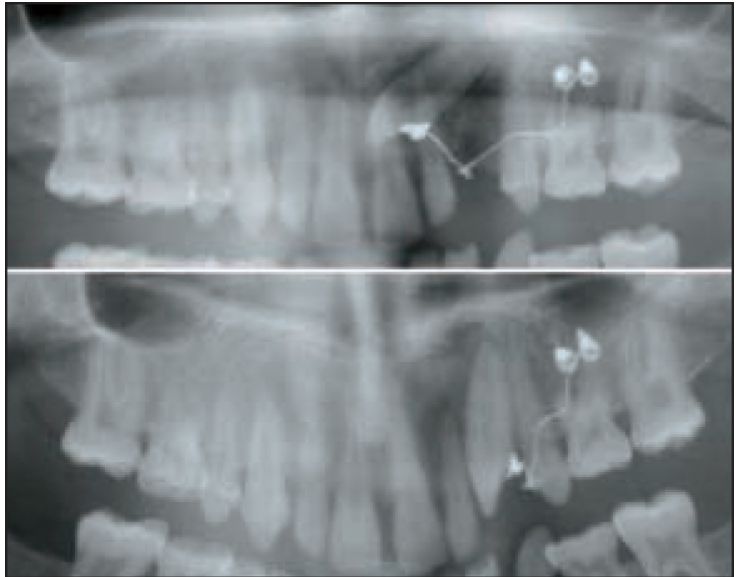

Figure $2 \mathrm{~g}$

Radiographs taken at a 14 month interval of the patient described above. The wire had propelled the impacted tooth into a satisfactory position without migrating under the mucosa.

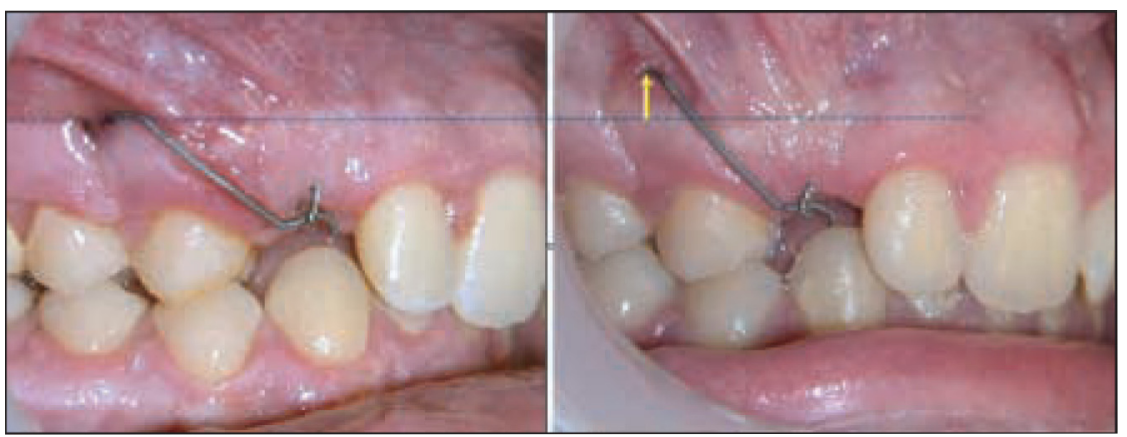

Figure $2 \mathrm{~h}$

The wire is exerting vertical pressure on a canine that is impacted in a high buccal position. The wire, which had emerged at the muco-gingival line, has progressively migrated under the mucosa thus impairing it efficiency.

it tighten up even more as it is activated. The alignment of the figure "8" loops is reversed depending on which side, left or right, or which arch, upper or lower, the device is destined to be set into (fig. $2 \mathrm{~d}$ and e).

The loops must be the right size, large enough not to be affected as the screws are being turned into place, and tight enough so they won't loosen after screws are fully set. This last risk can be completely obviated by placing a drop of solder on the loops with an electric welder.

When we first began to use this technique, we did not place the screws in the depth of the buccal cavity for fear of causing an infection and instead sought sites along the mucogingival line. This placement gave 

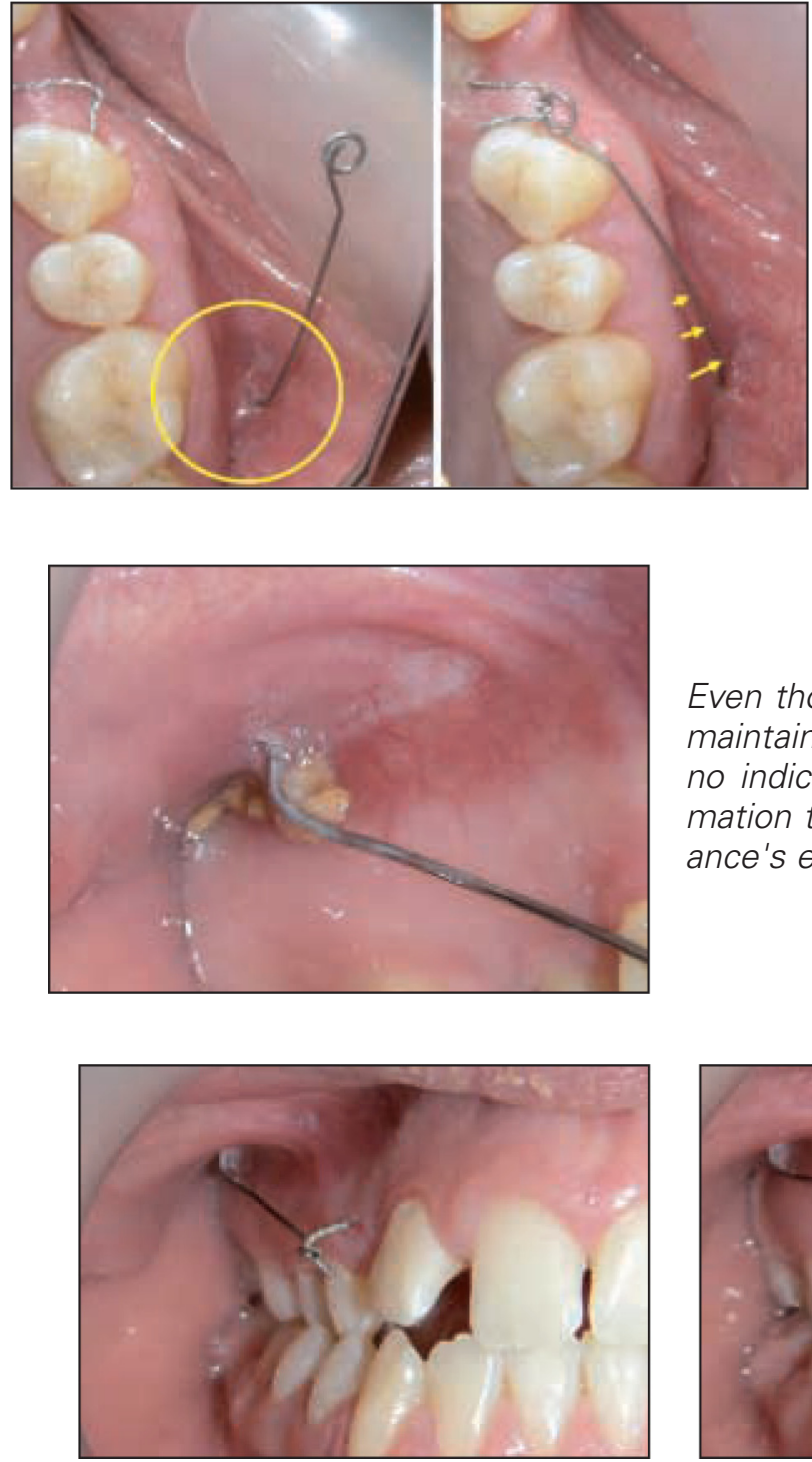

Figure $2 k$

As the impacted canine moves toward its correct position in the arch, the only adjustments that have to be made are shortening of the ligature wire that emerges from the gingiva.

excellent results when we were moving teeth buccally (fig. $2 \mathrm{f}$ and $\mathrm{g}$ ), but when we were moving teeth vertically it resulted in a migration of the embedded portion of the wire with a resultant loss of effectiveness in vertical traction (fig. 2 h). So we completely abandoned this type of

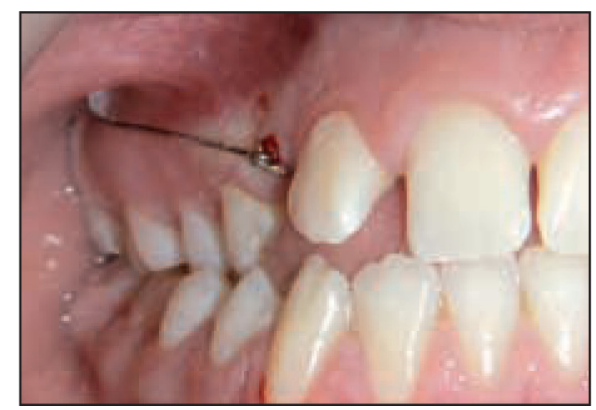

\section{Figure 2 i}

This wire emerges from the free mucosa at the center of the vestibule. Activated, itforms an angle of about $45^{\circ}$ with its rest position. When its attachment to the cuspid is tightened, it remains high enough above the gingival mucosa not to get embedded in it.

\section{Figure $2 j$}

Even though the patient has failed to maintain good oral hygiene, there is no indication of a developing inflammation that could decrease the appliance's effectiveness. 
reaction and they are hardly noticed by patients. Even in cases where patients lower their level of oral hygiene, we have not seen the development of any inflammations that could reduce the efficacy of the appliance (fig. 2 j).

We have noted that the quantity of force created at the free end of the wire is on the order of $65 \mathrm{cN}$.

The generous length of the free wire gives it a wide amplitude of move- ment without any loss of activation. The tension it expresses remains almost constant and there is usually no need for reactivation over the course of moving impacted teeth into place.

As the canines gradually approach their destination, the only adjustments we have to make are to shorten the ligature wire attached to the impacted tooth that emerges from the gingiva (fig. $2 \mathrm{k}$ ) and eventually to re adjust the direction of the force.

\section{3 - SURGICAL TECHNIQUE FOR SETTING THE WIRE IN PLACE BENEATH THE MUCOSA}

\section{3 - 1 - Operative protocol}

After the impacted canine has been uncovered and the traction wire attached to it, local anesthesia is injected near the zone where the screws will be placed, at a short distance from the site in order to reduce swelling. For upper canines the injection points will be mesial and distal to the zygomatic apophysis, previously located by palpation in relation to the upper first molar. For an impacted mandibular canine, the injection sites are mesial and distal to the lower first molar.

When anesthesia has been achieved, we make a horizontal incision of 10 to $12 \mathrm{~mm}$ with a scalpel at the depth of the vestibule deep enough to contact bone. Then we press the soft tissues away with a rugine to expose the bone where we have decided to place the screws.

To prepare for their insertion we pick up the first screw on the end of the screwdriver and set it into the mesial one of the traction wire's preformed loops. The operator holds this combination (fig. $2 \mathrm{c}$ ) with one hand, using the other hand to exert outward pressure on the patient's cheek to keep the incision opening sufficiently wide and also to avoid contaminating the screw as it is brought into the oral cavity and moved toward its destined position.

Depending upon where the impacted tooth is located the operator holds the free end of the activating wire in a defined fashion against the screwdriver, with the index finger for upper right and lower left canines and with the thumb for upper left and lower right impactions. This works out best for correct positioning of the wire (fig. 3 a).

Next the practitioner positions the screw against the bone and screws it in with enough pressure to insert it into the cortical bone (fig. 3 a). The wire, that had been free floating up to this point becomes fixed as the tightening 
screw engages it. Then the orthodontist loosens the screw slightly so the wire can be placed more precisely where desired. Then the practitioner picks up the second screw with the screwdriver and screws it firmly into place through the second loop and follows this by re-tightening the mesial screw so that now the traction wire is firmly in place against the bone surface. The dentist can perform these maneuvers without difficulty, even blind, because of the screw's attraction to the tip of the screwdriver.

The orthodontist then makes a bayonet bend in the traction wire with a ligature director assisted by finger pressure so that the wire will rise correctly from the bone and emerge into the center of the vestibule (fig. 2 i). The exact placement of the wire is important: too tight a contact with the gingiva could cause it to become embedded in mucosal tissue with a resultant limitation of the force it can develop (fig. 3 b). But if the wire

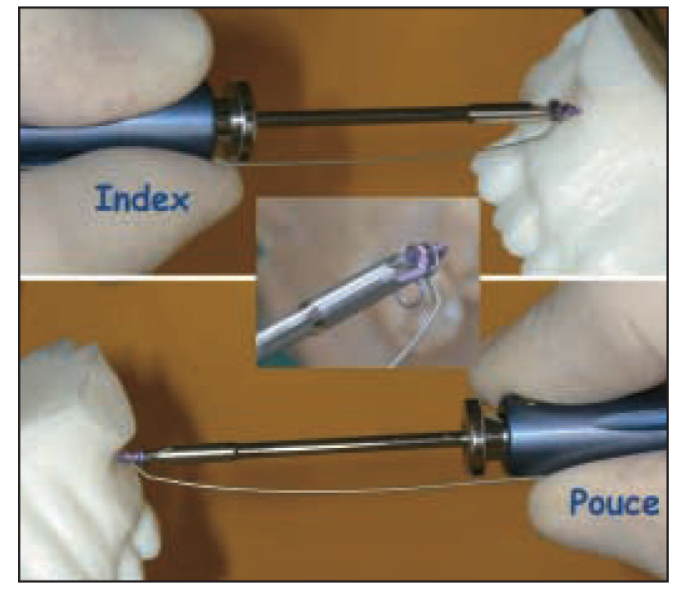

Figure 3 a

The orthodontist holds the free end of the wire with the index finger in setting up the appliance for upper right or lower left canines, and with the thumb in upper left or lower right impactions. These stances allow for correct wire placement. should be bent too far away from the gingiva it might irritate the cheek mucosa.

Next the orthodontist shapes the free portion of the wire with a Tweed pliers or ligature director to give it the desired shape and orientation, and, finally, bends a hook into its end. It will form an angle of about $45^{\circ}$ with the gingiva (fig. $2 \mathrm{i}$, fig. $3 \mathrm{c}$ and d).

The wire is then activated by ligating its end against the button attached to the impacted canine that is emerging from the gingiva at the very point of its destined position (fig. $3 \mathrm{c}$ and d).

\section{3 - 2 - Post-operative precautions}

Muscular tension from the cheeks and the lips will apply enough pressure to the borders of the incision to keep them approximated so that sutures are not necessary. Because of the minimum invasion of osseous

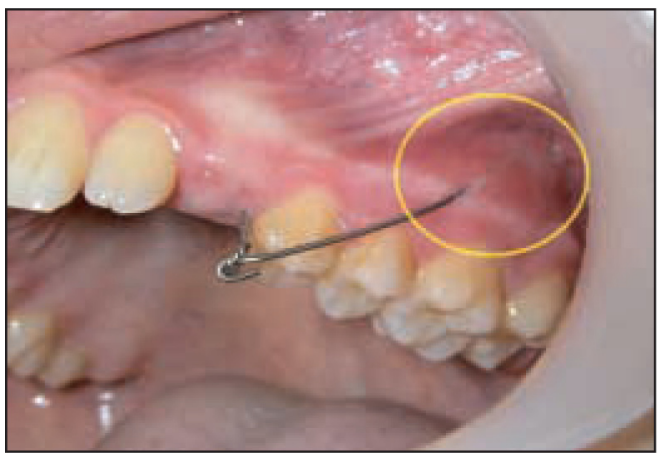

Figure $3 b$

The canine is moving in a satisfactory fashion as the increase in length of the ligature wire emerging from the gingiva testifies. However the too close contact of the CT8-1 wire with the gingival mucosa has buried part of the arch wire in tissue, thus risking its future efficiency. 

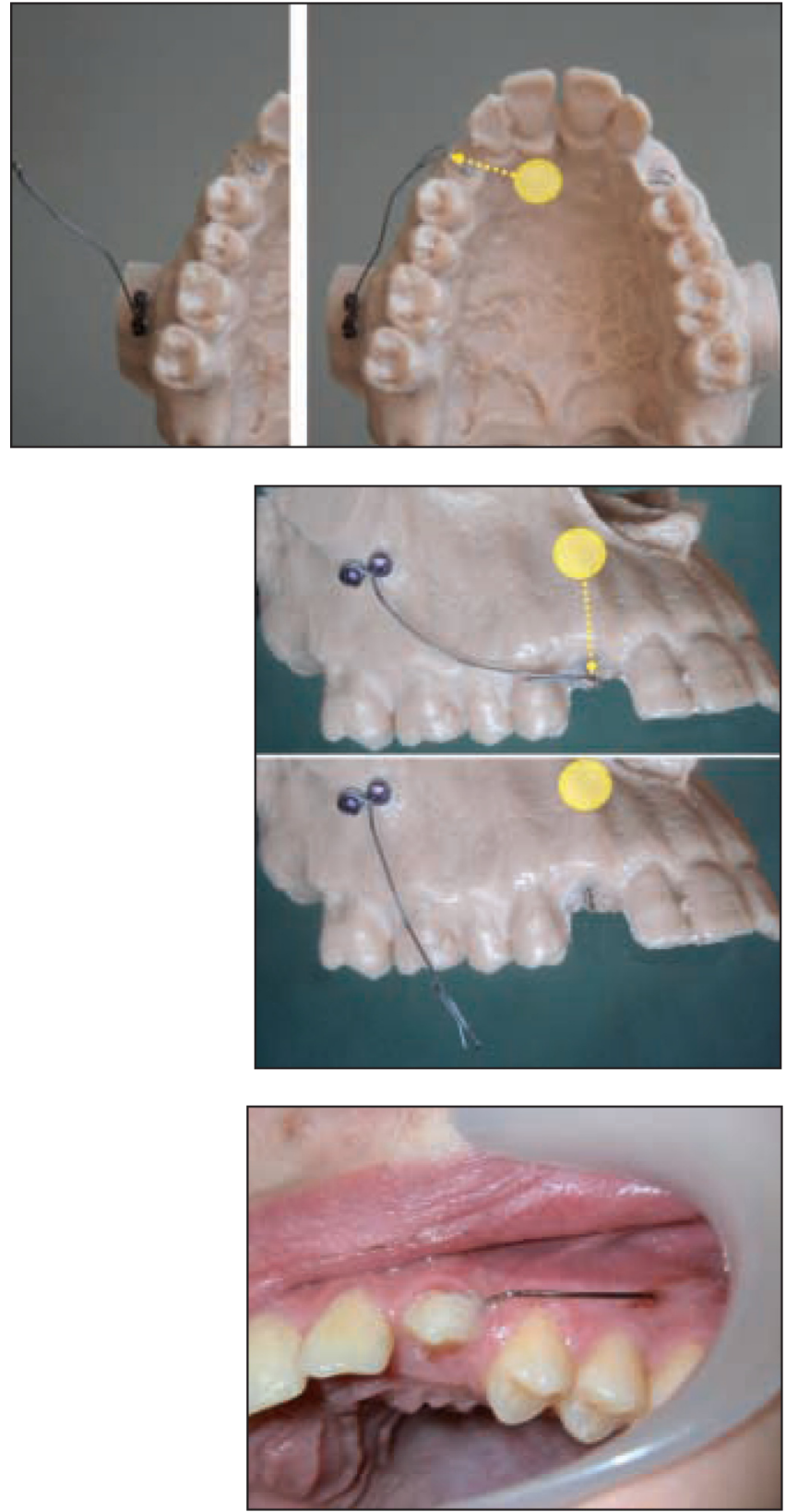

Figure 3 e

The end of the CT8-1 wire is bonded directly to the canine's crown.

tissue, post-operative complications are kept to a minimum. We limit our prescriptive dosage to a single type one analgesic and ask

\section{Figure $3 c$}

The free arm of the wire when activated to move a palatally impacted canine in a buccal direction forms an angle of about $45^{\circ}$ with its resting position. The orthodontist activates the wire by hooking its free end on the prepared ligature wire attached to the canine which emerges from the gingiva at the level of the tooth's future position.

\section{Figure $3 d$}

The free end of the wire, when activated, forms an angle of about $45^{\circ}$ with its nonworking position in order to move an impacted canine from its high buccal position in an occlusal direction. The orthodontists tenses it by hooking its terminal hook over a bulge prepared in the ligature wire attached to the canine which emerges from the gingiva at the level of the tooth's future position.

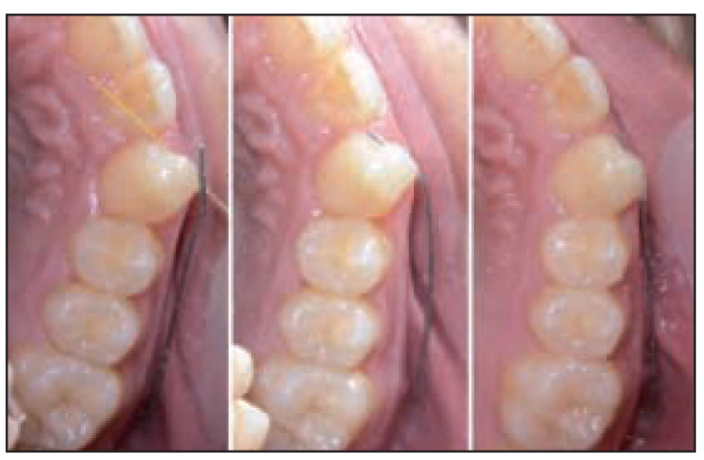

Figure $3 f$

The end of the wire rest in a composite tunnel made on the canine crown in order to correct its rotation.

our patients to cleanse the incision site with a 7/100 post-operative toothbrush dipped in a chlorhexidrine mouth wash. 


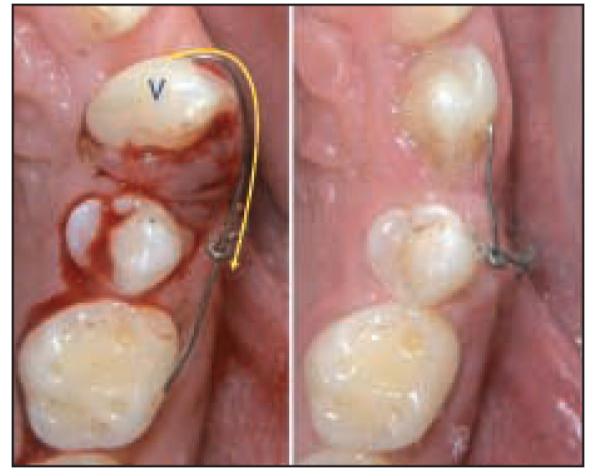

Figure $3 \mathrm{~g}$

A NiTi $.012(0,30 \mathrm{~mm})$ straight wire is bonded to the canine in order to correct a severe rotation. It is threaded into the CT8-1 where the sense of activation is reversed. It had been working in a buccal direction; now it will work in a palatal direction to initiate action of the NiTi wire, which, sliding freely in the loop, completely corrects the rotation as it resumes its original shape. The natural forces of occlusion prevent the tooth from moving lingually.

\section{3 - 3 - Treatment procedures}

The Tweed pliers and ligature directors are the only instruments we use to adjust the wire during the course of treatment.

We check the mechanism by unhooking the ligature wire (fig. 2 i). Usually the arch wire re-assumes the initial angle that it was given. Eventually its orientation can be adjusted as the canine moves closer to its designated position in the arch.

Activation usually consists of shortening or tightening the ligature wire as needed until the canine has made its appearance (fig. 2 k).

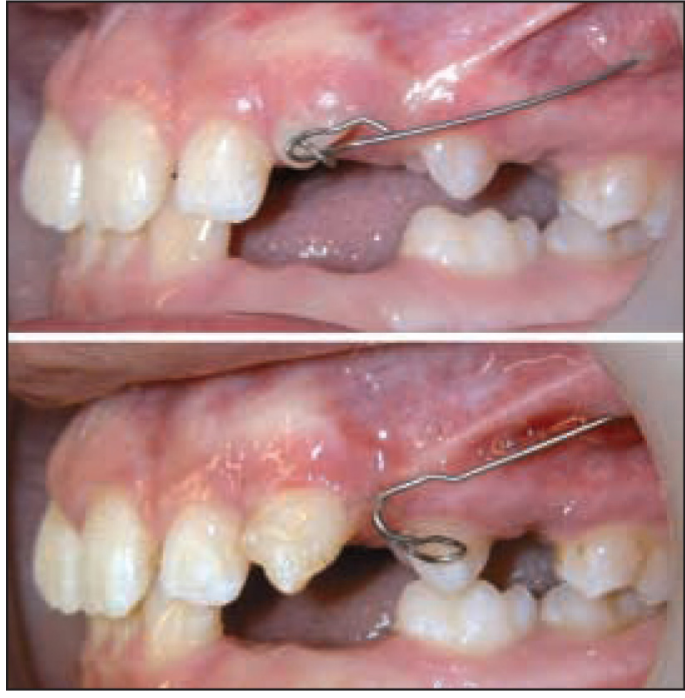

Figure $3 \mathrm{~h}$

An elastic chain has been bonded to the crown of the upper left canine and attached to a hook formed in the CT8-1 wire to move the tooth distally.

\section{3 - 4 - Finishing technique, correction of rotations}

When the impacted canine emerges enough from the gingiva to be accessible, the orthodontist can remove the button to which the ligature was attached and bond the activating wire itself directly to the tooth (fig. 3 e), set the wire to slide in a composite trench fabricated on the tooth (fig. $3 \mathrm{f}$ ) or, finally, brace it against a large stop on the tooth (fig. $4 \mathrm{~m}$ ).

The orthodontist can also bond another wire or an elastic chain in place as an adjunctive forces (fig. $3 \mathrm{~g}$ and h). Since it is possible to control the tension of the wire for all three 
dimensions of space, the orthodontist can usually move the impacted tooth in the appropriate direction and orientation without routinely having to incorporate other teeth in the set-up.

\section{3 - 5 - Removal of the appliance}

Removal of the screws and the CT8-1 wire poses no particular problems. All the orthodontist has to do is use a scalpel to make another incision similar to the first one, uncover the head of the screw with a rongeur or

\section{4 - A CLINICAL CASE}

Even though his temporary maxillary cuspids were still in place, it was discovered only at the relatively late age of 17 that this patient, Manuel, had palatally impacted canines (fig. 4 a). In all other respects, his occlusion could be considered satisfactory (fig. 4 b and c). After laying back a double palatal flap, we uncovered the canines and bonded buttons to their crowns (fig. 4 d). We did not need to open a mucosal window because the ligature wires attached to the buttons emerged at the sites of the temporary canines that we had extracted. We folded the ligature wires back, replaced the flap and sutured it.

Following the procedure already described, we made an incision on one side in the depth of the vestibule facing the first molar and screwed two short cortical screws in the base of the zygomatic process. They firmly held the CT8-1 wire which emerged through the incision, which we then bent into rugine, and apply the screwdriver to the screw's slot to unscrew it and remove it from the mouth. This can be done blind, by touch. The free end of the wire is then cut off at the gingiva level and the buried part removed through the incision.

There are no sutures to remove and there are virtually no post-operative problems. Our prescription remains the same, a type one analgesic as needed, and we ask patients to keep the site scrupulously clean with careful chlorhexydrine assisted brushing until healing is complete.

the desired shape with a Tweed pliers and a ligature director and then tied its extremity to the ligature attached to the canine button (fig. 4 e and $3 \mathrm{c}$ ).

We then repeated this operation for the tooth impacted on the other side (fig. 4 f), without placing any sutures. Finally, we took a check-up radiograph (fig. $4 \mathrm{~g}$ ).

Eight days later the quality of the healing in the vestibule can be seen in these views (fig. $4 \mathrm{~h}$ and i).

The only adjustments that we had to make during the subsequent treatment was to shorten the ligature wires as the canines moved further and further toward their end positions (fig. 4 j) and to control the direction of the traction force.

The upper left cuspid arrived at its place in the arch after three months of treatment (fig. $4 \mathrm{k}$ ) at which time we removed the button we had bonded to it when we uncovered it. We then bonded the traction wire directly to the tooth's crown so that we could achieve final detailed posi- 


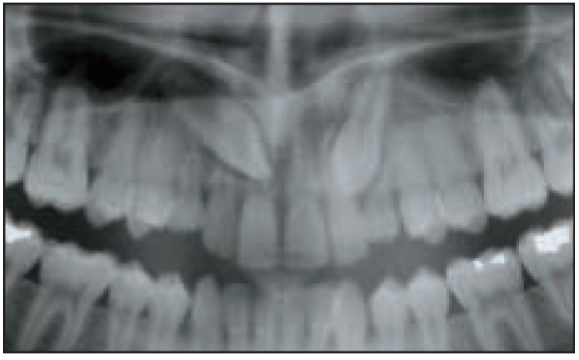

Figure 4 a

A panoramic film showing the two palatally impacted canines of a 17 year-old young man.

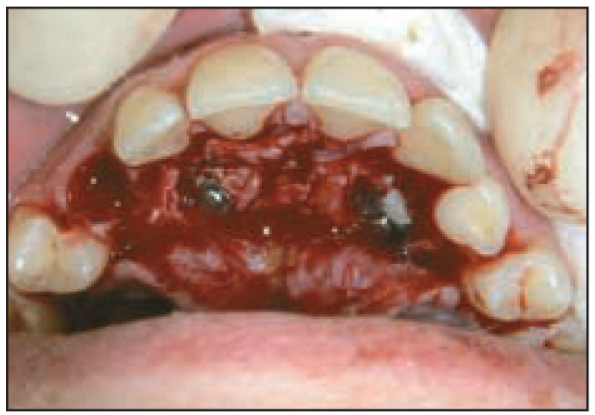

Figure $4 d$

With the aid of a double palatal flap, the canines were uncovered and buttons were bonded to their crowns.

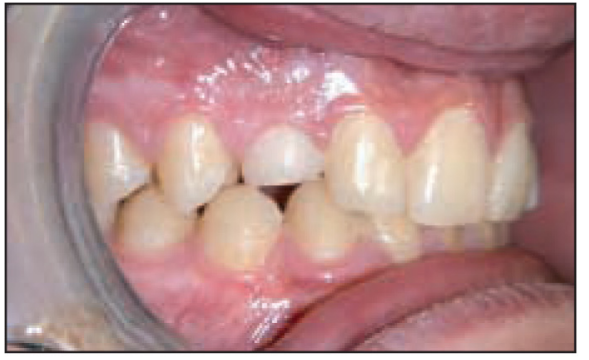

b

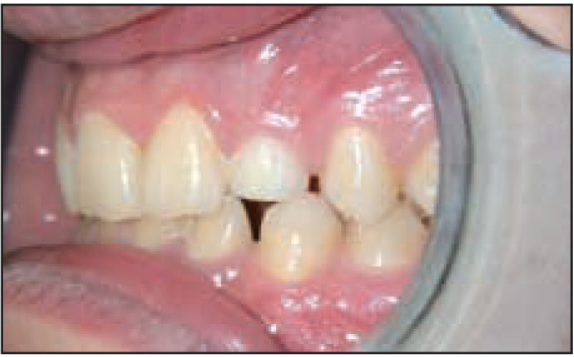

c

Figures $4 \mathrm{~b}$ and $c$

In other respects his occlusion is relatively satisfactory.
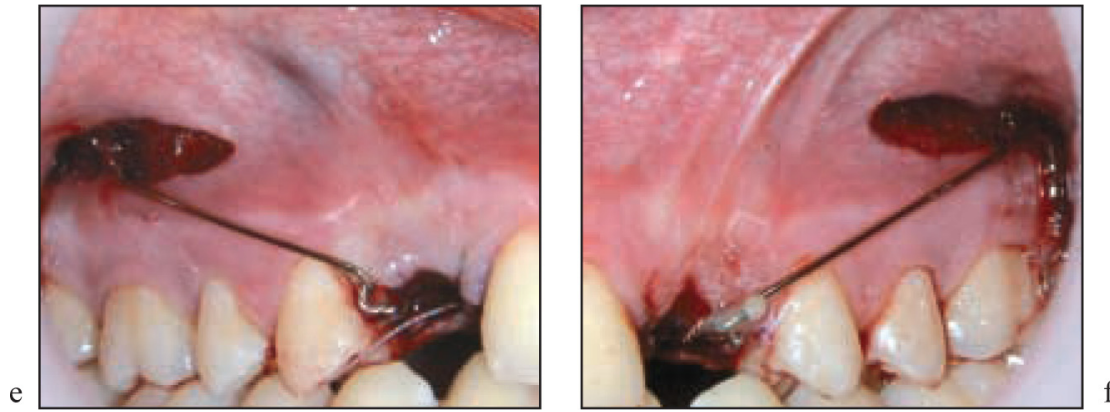

Figures 4 e and $f$

Al CT8-1 wire, held by two screws, emerges through the incision. After having been activated buccally, the terminal end of the wire is hooked to a ligature tied to the impacted canine. Pressure from a lip retractor is keeping the incision open. It's borders will approximate when the retractor is removed and remain closed from natural muscular pressure, obviating need for suturing.

$\mathrm{h}$

Figure $4 \mathrm{~g}$

A check-up radiograph taken immediately after the procedure.
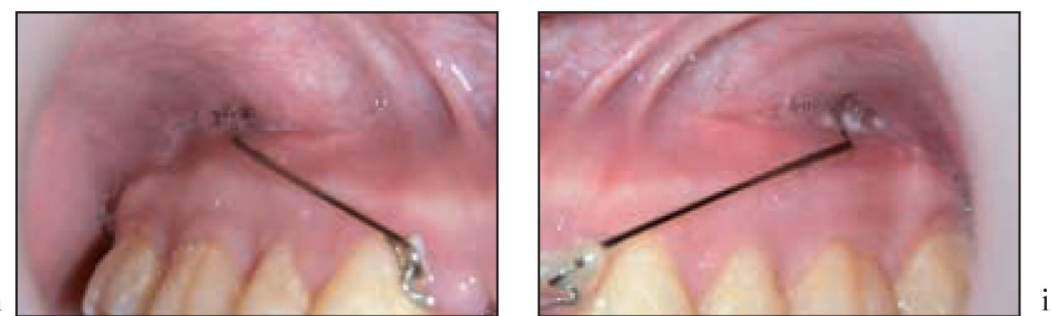

Figures $4 h$ and $i$

Eight days later, healing of the buccal tissue is satisfactory. 


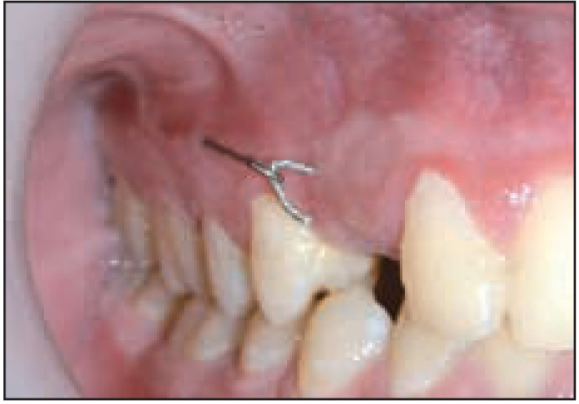

Figure $4 j$

The canine has moved buccally thanks to traction from the CT8-1 wire. The only adjustments we had to make were progressively to shorten the ligature wire attached to the canine crown.

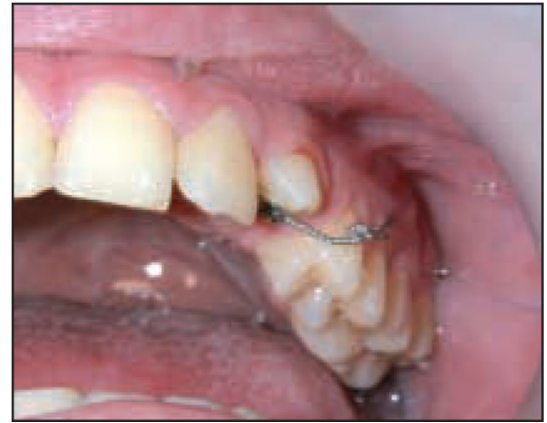

Figure $4 k$

When the upper left canine emerged into the oral cavity after three months of treatment, we removed the palatal button.

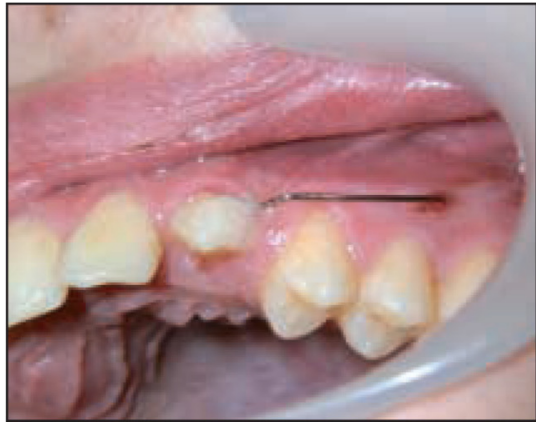

Figure 41

We then bonded the wire directly to the tooth's crown to complete its positioning in the arch.
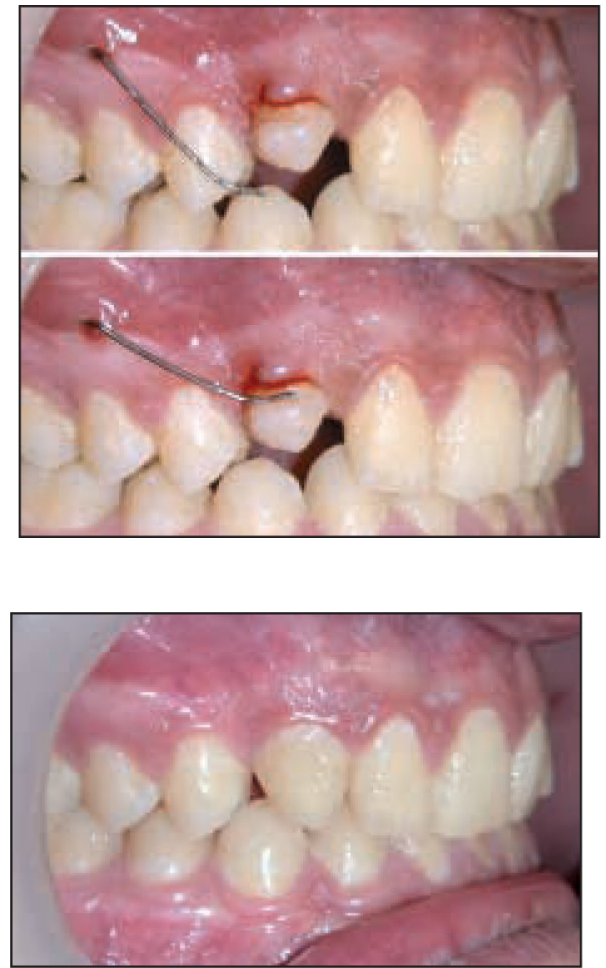

Figure $4 n$

Right side view at the end of treatment showing the canine in correct position.
Figure $4 \mathrm{~m}$

When the upper right cuspid appeared, its button was also removed. We then bonded a composite stop on the crown's buccal surface for the wire to butt against and finish moving the tooth into place. Had we bonded the wire directly to the tooth, instead of letting it slide freely, the wire's activation would have imparted an undesirable distal component of force to the system.

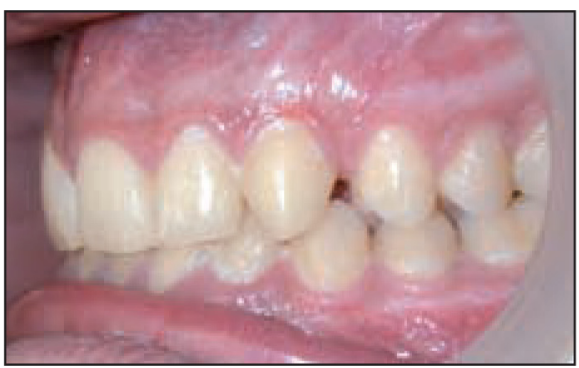

Figure 40

Left side view at the end of treatment showing the canine in correct position. 


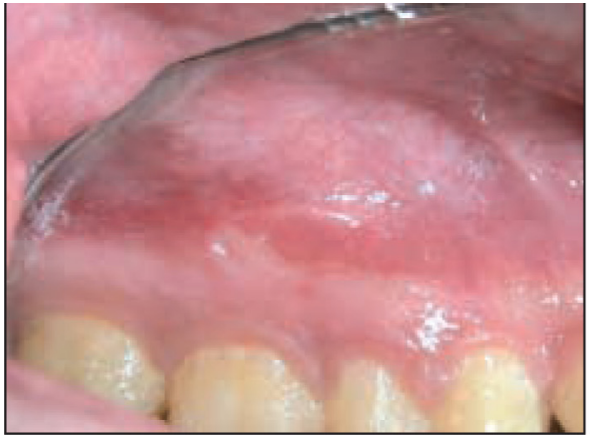

Figure $4 p$

A week after removal of the screws and the CT8-1: the gingiva on the right side has completely healed showing no traces of the procedures it had endured.

tioning (fig. 4 l). As soon as it emerged, we removed the upper right canine's button and fixed a small composite wedge on its crown so that we could apply pure egressive force to it (fig. $4 \mathrm{~m}$ ). We did not bond the wire directly to the tooth because that might have imparted an unwanted distal component of force to the traction.

\section{5 - CONCLUSION}

This simple mechanism, consisting of two short cortical screws placed under the mucosa at the depth of the vestibule and a TMA wire emerging at a distance, is all that is needed to move an impacted canine completely into position. It requires the involvement of no other teeth for anchorage, making it an entirely independent apparatus needing the support of no additional appliances or dental units.

In contrast to other mechanical systems relying on trans-mucosal

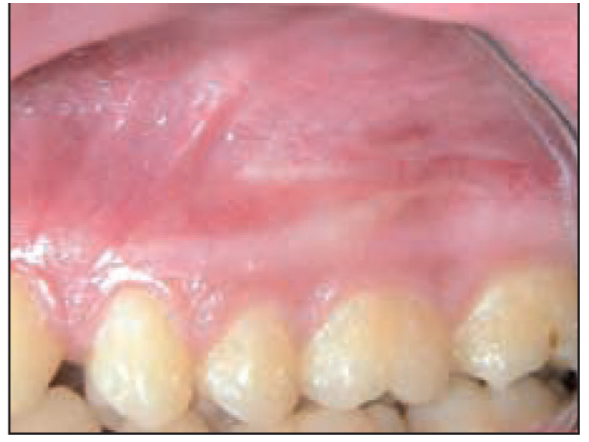

Figure $4 \mathrm{q}$

A week after removal of the screws and the CT8-1: the gingiva on the left side has completely healed showing no traces of the procedures it had endured.

When both teeth were in their correct positions we removed the entire apparatus (fig. $4 \mathrm{n}$ and o). One week later there were no traces in the mucosa of the operations that had been performed (fig. $4 \mathrm{p}$ and q). The successful treatment had been extremely simple and straightforward, requiring very few activations and no wire changes. It lasted for 13 months.

mini-screws, this mechanism can always be used because it does not depend on the quality of available bone or keratinized gingiva, or have to avoid impinging on nearby roots. It is perfectly reliable and incurs no risk of the screws becoming loose. Any practitioner can install it, risk free and with very few post-operative complications, which is not the case with embedded plates.

To sum up, we are presenting a simple system that is reliable, autonomous, reproducible and polyvalent. We have shown how it 
can be used to move impacted canines into their correct positions in the arch but many other indications for it exist. It is a technique of the future that only requires further refinement.

\section{REFERENCES}

1. Amat P. Entretien avec Hugo De Clerck. Rev Orthop Dento Faciale 2006;40:9-36.

2. Asscherickx $K$, Vannet $B V$, Wehrbein $H$, Sabsevar MM. Root repair after injury from miniscrew. Clin Oral Implants Res 2005;16:575-8.

3. Chillès $D$. Ten years using maxillofacial surgical screws as orthodontic anchorage. $6^{\text {th }}$ International Orthodontic Congress World J Orthod 2005;6(suppl.):114.

4. Chillès $D$, Chillès J.-G. Introduction à I'utilisation de vis de chirurgie maxillo-faciale comme ancrage orthodontique. Rev Orthop Dento Faciale 2006;40:63-90.

5. Chillès D. L'ingression incisive grâce aux mini vis corticales. Journées de I'Orthodontie de la Fédération, Paris, 10/11/2008.

6. Cuinet M, Guivarch J, Huet AP, Morgon L. Les différents alliages utilisés en orthodontie. Orthod Fr 2001;72:271-8.

7. De Clerck H, Geerinckx V, Siciliano S. The Zygoma Anchorage System. J Clin Orthod 2002;36(8):455-9.

8. Kravitz ND, Kusnoto B. Risks and complications of orthodontic miniscrews. Am J Orthod Dentofacial Orthop 2007;131 S:43-50.

9. Lee JS, Kim JK, Park YC, Vanarsdall Jr RL: Applications cliniques des mini-implants en orthodontie. Quintessence édit 2007.

10. Maino BG, Maino G, Mura P. Spider Screws: skeletal anchorage system. Prog Orthod 2005;6(1):70-81.

11. Melsen B. Indications d'ancrage squelettique en orthodontie. Rev Orthop Dento Faciale 2006;40:41-61.

12. Miyawaki S, Koyama I, Inoue M et coll. Factors associated with the stability of titanium screws placed in the posterior region for orthodontic anchorage. Am J Orthod Dentofacial Orthop 2003;124:373-8.

13. Sugawara J. Objectifs et sécurité dans l'orthodontie de l'adulte grâce aux nouveaux Systèmes d'Ancrage Squelettique (SAS). S.F.O.D.F., Paris 28/01/2007. 